\title{
Ultrasensitive liquid level sensor based on slice-shaped composite long period fiber grating
}

\author{
Xiren Jin (金夕人) ${ }^{1}$, Zeju Rui (芮泽钜) ${ }^{1}$, Zihang Xiang (向子航) ${ }^{1}$, Chupeng Lu (卢楚鹏) ${ }^{1}$, Shuo Zhang (张 硕) ${ }^{1}$, \\ Xian Xu (徐 显) $)^{1}$, Mingyang Lü (吕明阳) ${ }^{1}$, Yiwei Ma (马一巍) ${ }^{1}$, Cuiting Sun (孙翠婷) ${ }^{2}$ ， Xinghua Yang (杨兴华) ${ }^{1}$, \\ Tao Geng (耿 涛) $)^{1^{*}}$, Weimin Sun (孙伟民) $)^{1 * *}$, and Libo Yuan (苑立波) ${ }^{3}$ \\ ${ }^{1}$ Key Laboratory of In-fiber Integrated Optics, Ministry of Education, College of Physics and Optoelectronic Engineering, Harbin Engineering University, \\ Harbin 150001, China \\ ${ }^{2}$ Acoustic Science and Technology Laboratory, College of Underwater Acoustic Engineering, Harbin Engineering University, Harbin 150001, China \\ 3 Photonics Research Center, Guilin University of Electronics Technology, Guilin 541004, China
}

*Corresponding author: gengtao_hit_oe@126.com

${ }^{*}$ Corresponding author: sunweimin@hrbeu.edu.cn

Received July 30, 2021 | Accepted September 15, 2021 | Posted Online October 25, 2021

\begin{abstract}
In this paper, a novel liquid level sensor with ultra-high sensitivity is proposed. The proposed sensor is configured by a sliceshaped composite long period fiber grating (SSC-LPFG). The SSC-LPFG is prepared by polishing two opposite sides of a composite multimode-single-mode-multimode fiber structure using a $\mathrm{CO}_{2}$ laser. The method improves the sensitivity of the sensor to external environment. Based on the simulation calculation, a liquid level sensor with a length of $3 \mathrm{~mm}$ is designed. The experimental transmission spectrum agrees well with the simulation result. The experimental results show that the sensitivity reaches $7080 \mathrm{pm} / \mathrm{mm}$ in the liquid level range of $0-1400 \mu \mathrm{m}$ in water. The temperature sensitivity is $24.52 \mathrm{pm} /{ }^{\circ} \mathrm{C}$ in the range of $20^{\circ} \mathrm{C}-90^{\circ} \mathrm{C}$. Due to the ultra-high sensitivity, good linearity, and compact structure, the SSC-LPFG has potential application in the field of high-precision liquid level measurement.
\end{abstract}

Keywords: liquid level sensor; composite long period fiber grating; ultra-high sensitivity.

DOI: 10.3788/COL202220.011202

\section{Introduction}

Optical fiber sensors have the advantages of strong resistance to electromagnetic interference, high sensitivity, no operating current, and ability to work in complex environments ${ }^{[1]}$. Therefore, they are widely used in temperature ${ }^{[2]}$, refractive index $(\mathrm{RI})^{[3]}$, bending $^{[4]}$, strain ${ }^{[5,6]}$, liquid level ${ }^{[7]}$, and other measurements ${ }^{[8]}$, especially in chemistry and biotechnology applications ${ }^{[9]}$. Among them, liquid level is an important parameter in chemical industry production, fuel storage, water level monitoring, medical testing, dose control, and biochemical progress fields. In some specific environments, for example, aircraft fuel level monitoring in the aviation field ${ }^{[10]}$, the quantitative measurement of water infiltration in the underwater launch chamber ${ }^{[11]}$, and the liquid probe in the medical field ${ }^{[12]}$, high-precision and small-range liquid level sensors are needed, so it is of great significance to carry out research on small-range liquid level sensors. In environments that require ultra-high liquid level sensitivity like medical testing, the common capacitance, resistance, magnetostriction, and ultrasonic sensors have various defects and cannot meet the requirements of liquid level measurement ${ }^{[13]}$. In recent years, various optical fiber liquid level sensors were proposed, such as fiber Bragg gratings (FBGs) ${ }^{[14,15]}$, long period fiber gratings $\left(\right.$ LPFGs $^{[16,17]}$, Michelson interferometers (MIs) ${ }^{[18]}$, and MachZehnder interferometers (MZIs) ${ }^{[19,20]}$. Especially, the sensor based on a multimode fiber (MMF) structure has attracted more and more attention because of its advantages such as simple structure, low cost, and convenient manufacture. Because more modes and fields leak into its surroundings providing stronger lightmatter interaction, the sensor will be more susceptible to the influence of external environment changes. Thus, the sensor based on a multimode structure has shown great potential in the measurement of liquid levels.

In the research of high-sensitivity liquid level measurement, some people proposed the MMF cascading hollow-core fiber (HCF) and FBG (SMF-MMF-HCF-SMF/FBG) with an aligned spliced structure ${ }^{[21]}$, surrounding RI (SRI)-based etched chirped FBG structure ${ }^{[22]}$, and MMF-HCF-FBG structure ${ }^{[23]}$. MMF has many modes and strong RI modulation ability, which can effectively shorten the grating length. In the process of SMF and MMF coupling, higher-order modes can be excited to improve sensor performance. We use polishing to enhance 
the evanescent field on the surface of the optical fiber, making the sensor more sensitive to the external environment. Compared with these structures, in this paper, we propose an ultra-high-sensitivity sensor for liquid level measurement. The sensor has high liquid level sensing sensitivity and low temperature crosstalk. The proposed sensor is configured by a sliceshaped composite LPFG (SSC-LPFG), in which the liquid level sensitivity reaches $7080 \mathrm{pm} / \mathrm{mm}$. The temperature sensitivity is $24.52 \mathrm{pm} /{ }^{\circ} \mathrm{C}$. Compared with the MZI sensors, the SSC-LPFG achieves higher sensing characteristics without increasing the complexity of preparation. Its level sensitivity is much higher than that of most known level sensors in the measurement range of $1.4 \mathrm{~mm}$. Meanwhile, we simulate the energy transfer diagram and the transmission spectrum, and the simulation results are consistent with the experimental results. Due to the advantages of ultra-high sensitivity, compact structure, and good stability, it has potential application in the detection of liquid levels of medical, industrial, and other fields.

\section{Fabrication and Principle Analysis}

The SSC-LPFG is fabricated by polishing on the composite multimode-single-mode-multimode (C-MSM) fiber structure. We use three precision displacement platforms (accuracy is $0.01 \mathrm{~mm}$ ) to fix the fiber and a fiber cleaver to cut the fiber, using a microscope with CCD for monitoring the cutting fiber length. The specific process is as follows. Firstly, the C-MSM section is fabricated by using an optical fiber cutter (FC-6S) and a fusion optical fiber splicer (Fujikura, 86C). A conventional segment of SMF is spliced to an MMF. After that, the part of a sensing element (MMF) is cleaved, and another segment of SMF is spliced again. As the MMF length is $200 \mu \mathrm{m}$, the structure will be more sensitive to changes in the external environment ${ }^{[24]}$. In order to meet the phase matching conditions and get better sensing characteristics, the specific lengths of the cutting MMF and SMF are $200 \mu \mathrm{m}$ and $500 \mu \mathrm{m}$, respectively. The above splicing process is repeated five times to fabricate the C-MSM structure.

Secondly, the high-power $\mathrm{CO}_{2}$ laser is used to polish on the C-MSM structure in the two opposite directions parallel to the fiber axis. The schematic diagram of $\mathrm{CO}_{2}$ laser polishing is shown in Fig. 1. The plane depth is $30 \mu \mathrm{m}$ on the first side to ensure more exposure to the evanescent field. Then, the polishing step is repeated after the sample is twisted $180^{\circ}$.

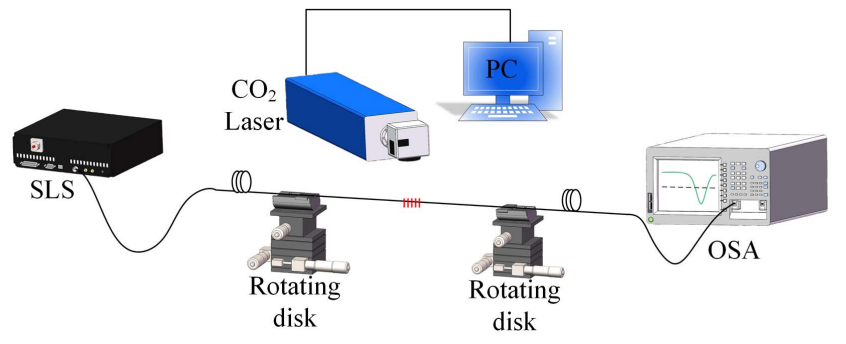

Fig. 1. Experimental device diagram for fabricating the SSC-LPFG.

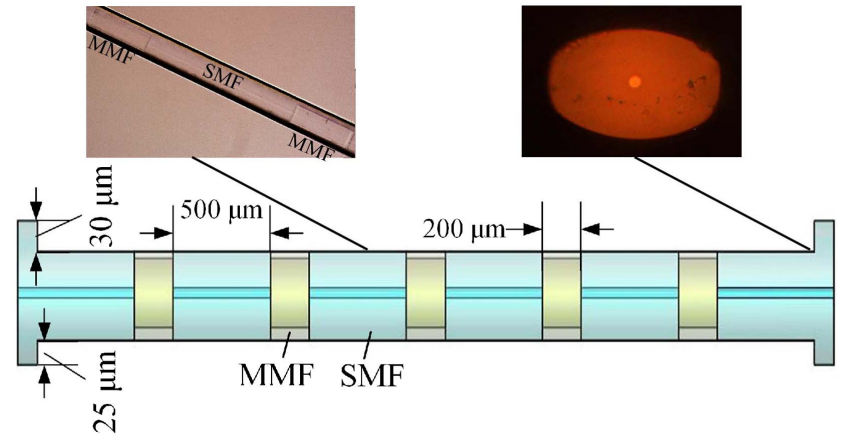

Fig. 2. Scheme diagram of the SSC-LPFG fiber structure.

The fabrication is completed when a sufficiently deep resonant dip appears at $1492 \mathrm{~nm}$. The obtained structure is slice-shaped, so we name it SSC-LPFG. Figure 2 shows the scheme diagram of the SSC-LPFG. The total length of the SSC-LPFG is $3 \mathrm{~mm}$. The experimental transmission spectrum is shown as the blue line in Fig. 3(b).

We use the R-soft simulation software to simulate the energy transfer diagram and the transmission spectrum. In the R-soft simulation process, the core/cladding diameters of the MMF and SMF are $60 / 125 \mu \mathrm{m}$ and $8 / 125 \mu \mathrm{m}$, respectively. The mesh size is $0.1 \mu \mathrm{m}$ in the $X Y$ direction and $0.4 \mu \mathrm{m}$ in the $Z$ direction. We set the SMF cladding RI to 1.4468 , the core RI to 1.4521 , the MMF cladding RI to 1.4781 , and the core RI to 1.4783 . Figure 3(a) shows that the energy in the core is gradually coupled to the cladding as the light propagates in the structure. Figure 3(b) shows the experimental and simulated transmission spectra of the SSC-LPFG structure, and the simulation results are consistent with the experimental results.

The phase matching condition satisfies the following relationship ${ }^{[25]}$ :

$$
\lambda=\left(n_{\mathrm{core}}^{\mathrm{eff}}-n_{\mathrm{cl}, k}^{\mathrm{eff}}\right) \cdot \Lambda,
$$

where $\lambda$ is the wavelength, $n_{\text {core }}^{\text {eff }}$ and $n_{\mathrm{cl}, k}^{\text {eff }}$ are the effective RIs of the core mode and the $k$ th diffraction cladding mode, and $\Lambda$ is the period of the grating. The minimum transmission value and width of the resonant dips are determined by the coupling
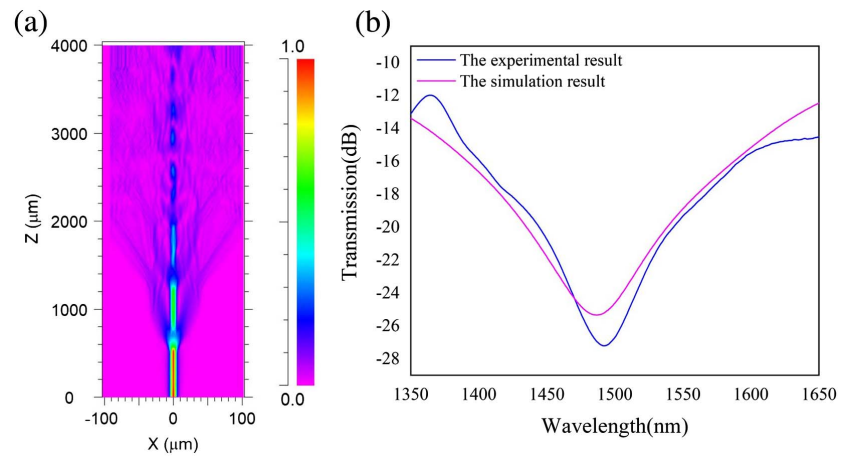

Fig. 3. (a) Simulated energy transfer diagram. (b) Experimental and simulated transmission spectra of the SSC-LPFG structure. 


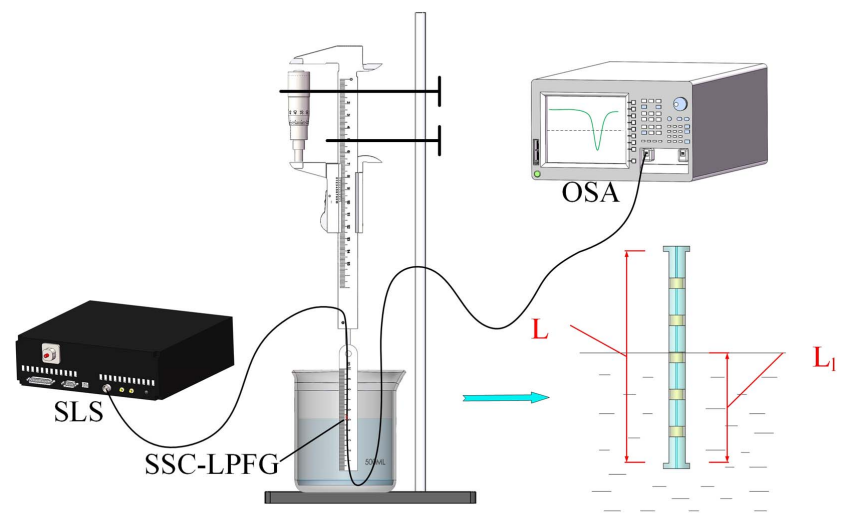

Fig. 4. Liquid level measuring device.

efficiency between the core and the cladding modes and the length of the LPFG. The minimum transmission value of the resonant dips is related to the length of the LPFG as ${ }^{[26]}$

$$
T(L)=\cos ^{2}(\kappa L)
$$

where $L$ is the length of the LPFG, and $\kappa$ is the coupling coefficient. The RI sensitivity of the central wavelength of the resonant dips of the LPFG arises from the dependence of the cladding modes' effective RI on the RI of the surrounding material. These effects permit the use of an LPFG as an RI sensor based on changes in the wavelength or the minimum transmission value of the resonant dips in the LPFG spectrum.

The RI sensitivity is used to measure the concentration of some substances ${ }^{[27]}$, so we use it to measure the liquid level. When the SSC-LPFG is partially immersed within the liquid, it can be considered to be two separate LPFGs. As shown in Fig. 4, where $L_{1}$ is the LPFG length surrounded by liquid, $L-L_{1}$ is the LPFG length surrounded by air, and $L$ is the length of the overall LPFG. For each cladding mode, the transmission spectrum will contain two resonant dips, as shown in Fig. 5. One is centered

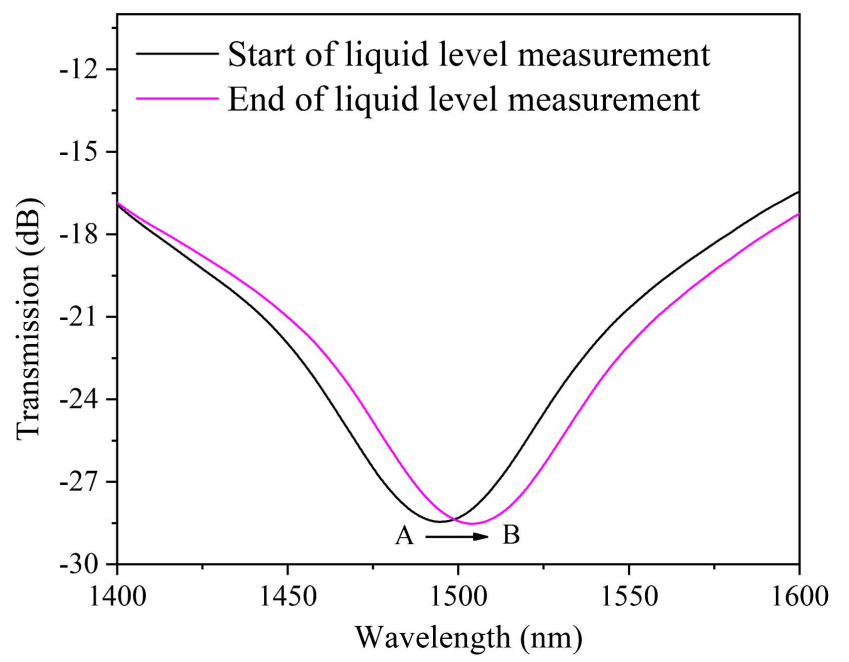

Fig. 5. Liquid level measurement begins and ends with wavelength drift. at the coupling wavelength of the result where the minimum transmission value and width of the resonant dips will depend on the liquid level. By using Eq. (2), for an LPFG of length $L$, it can be shown that the dependence of the minimum values of resonant dips $A$ and $B$, on the length of the LPFG immersed in the liquid $L_{1}$, is given by

$$
\begin{gathered}
T_{A}\left(L_{1}, L\right) \propto \cos ^{2}\left[\frac{\pi}{2}\left(\frac{L-L_{1}}{L}\right)\right], \\
T_{B}\left(L_{1}, L\right) \propto \cos ^{2}\left[\frac{\pi}{2}\left(\frac{L_{1}}{L}\right)\right],
\end{gathered}
$$

where $T_{A}$ and $T_{B}$ are the minimum transmission values of resonant dips A and B, respectively. Equations (3) and (4) are valid in the range of $0<L_{1}<L$. From Eqs. (3) and (4), if $L_{1}=0$, $T_{A}$ reaches the minimum, $T_{B}$ reaches the maximum, and the superimposed resonant dip consisting of resonant dips $A$ and $B$ coincides with resonant dip A. As the liquid level increases, $T_{A}$ monotonously increases, $T_{B}$ monotonously decreases, and the superimposed resonant dip gradually shifts from the wavelength of resonant dip A to that of resonant dip B. If $L_{1}=L, T_{A}$ reaches maximum, $T_{B}$ reaches minimum, and the superimposed resonant dip coincides with resonant dip B. Both $T_{A}$ and $T_{B}$ are monotonic functions, so the changes of $T_{A}$ and $T_{B}$ are always opposite, and the shift of the superimposed resonant dip is unidirectional. Thus, the proposed sensor can measure the liquid level change.

\section{Experimental Results and Discussion}

The experimental device of the SSC-LPFG for liquid level measurement is shown in Fig. 4. The spiral micrometer pushes the connecting rod down and into the water. The moving distance of the spiral micrometer rod is the same as that of the sensor in the liquid. Figure 6 shows that the levels change from $0 \mu \mathrm{m}$ to $1400 \mu \mathrm{m}$, and the wavelength shift is recorded at the same time

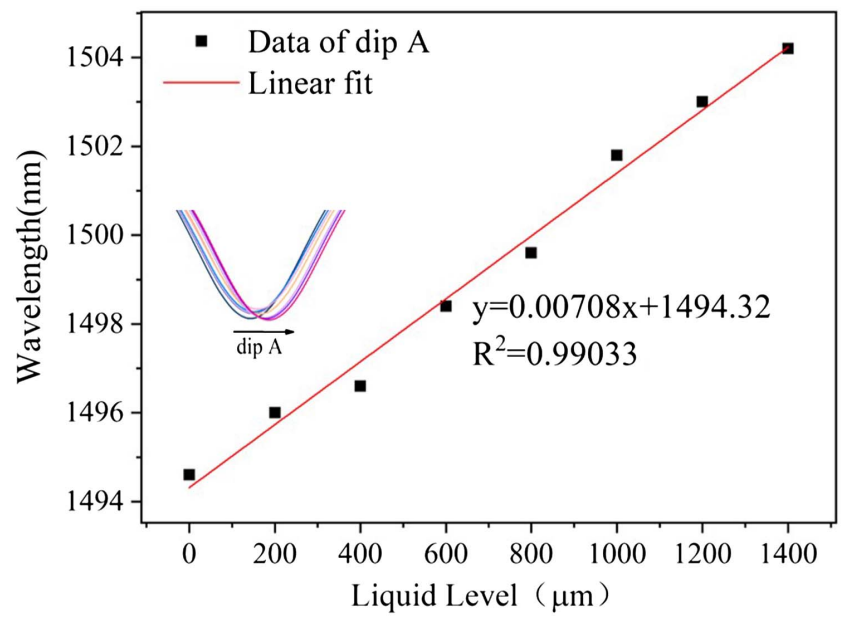

Fig. 6. SSC-LPFG liquid level sensing performance at different liquid levels. 
every $200 \mu \mathrm{m}$. As the liquid level increases, the wavelength of the resonant dip will have a red shift from $1494.6 \mathrm{~nm}$ to $1504.2 \mathrm{~nm}$. The sensitivity of the water level measurement is $7080 \mathrm{pm} / \mathrm{mm}$, which is described by linear fitting with a correlation coefficient $\left(R^{2}\right)$ of 0.99033 .

To compare the liquid level performance of different polishing depth sensors, we made three sensors with polishing depths of $15 \mu \mathrm{m}, 20 \mu \mathrm{m}$, and $25 \mu \mathrm{m}$ on the second side and compared their liquid level sensing performance. Figures $7(a)$ and $7(b)$, respectively, show the resonant dip with three different polishing depths and liquid level sensing performance of sensors. The $R^{2}$ of the sensor with a polishing depth of $15 \mu \mathrm{m}$ is 0.93966 , which has low linearity and cannot accurately measure the liquid level. The sensor with polishing depth of $20 \mu \mathrm{m}$ has a liquid level sensitivity of $2930 \mathrm{pm} / \mathrm{mm}$ and $R^{2}$ of 0.87226 . The SSC-LPFG in this paper has a level sensitivity of $7080 \mathrm{pm} / \mathrm{mm}$ and $R^{2}$ of 0.99033. The experimental results show that the proposed SSC-LPFG has the best sensing performance.

The liquid level performance of SSC-LPFG is repeatedly measured two times to verify stability. The liquid level sensing
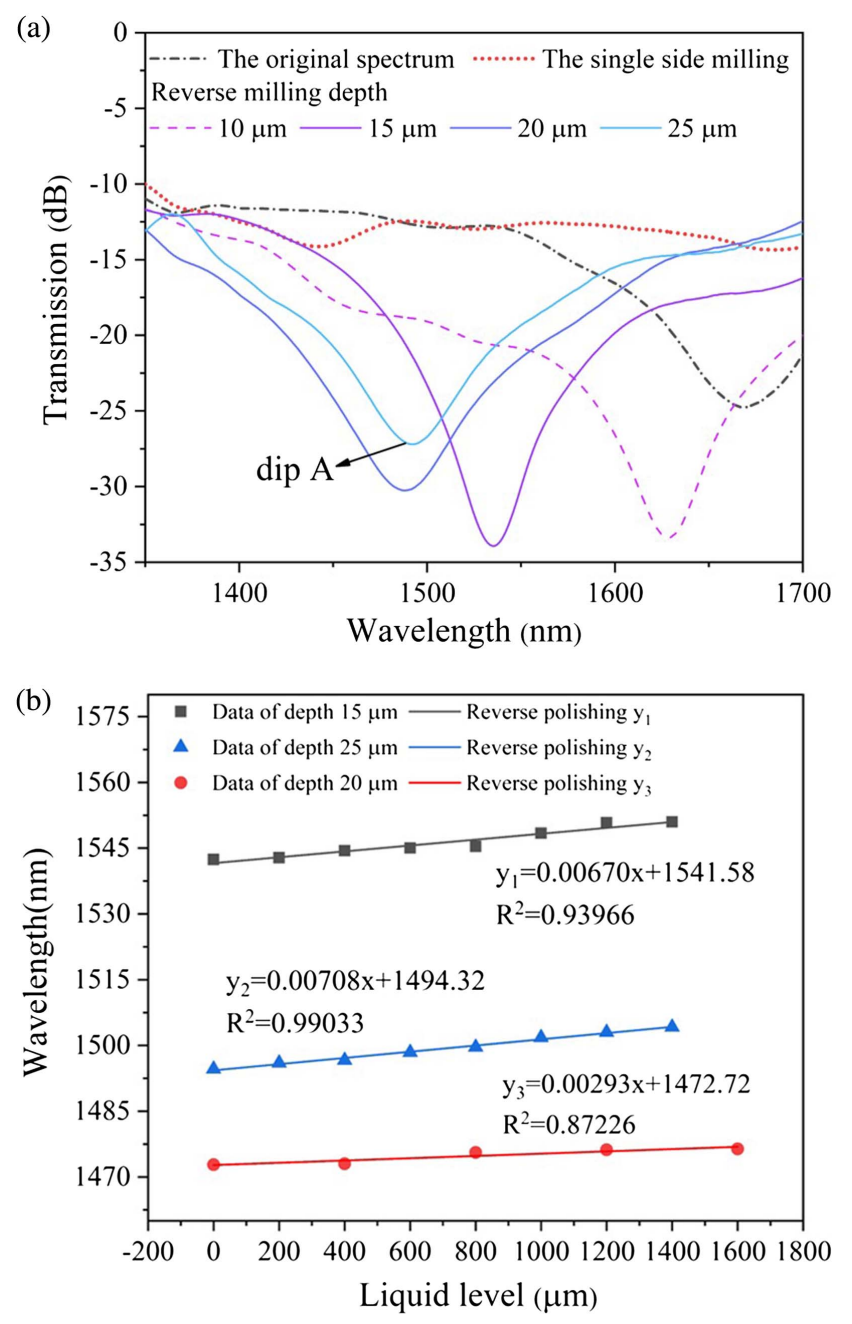

Fig. 7. (a) Resonant dip of sensors with different polishing depths. (b) Dip wavelength shift versus liquid level (different depth of polishing].

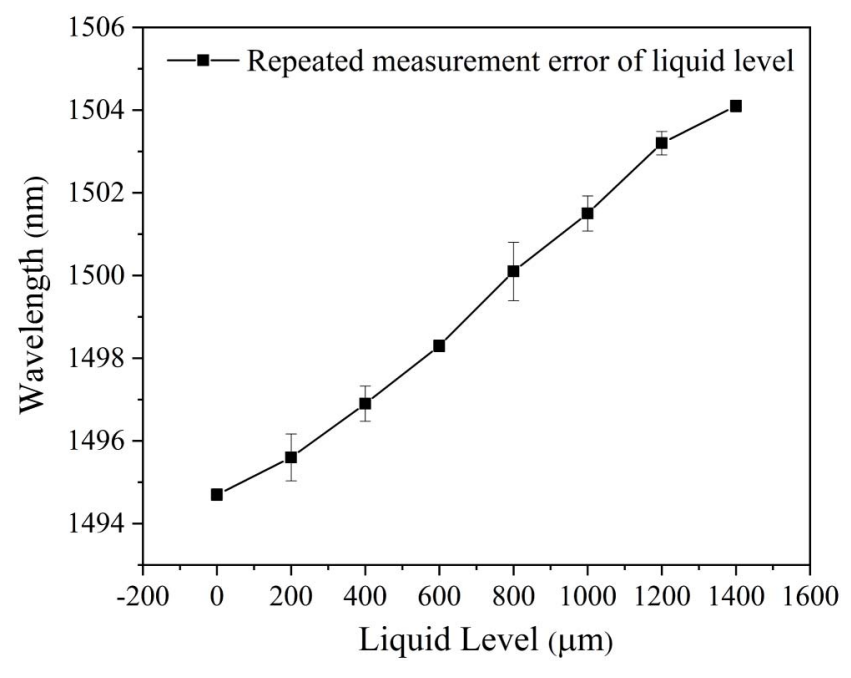

Fig. 8. Liquid level sensing performance.

performance is shown in Fig. 8. It shows that SSC-LPFG has stable performance in liquid level measurement. At the same time, we measure the temperature sensitivity of SSC-LPFG by exposing it to air. The sensor is placed into the temperature control platform, which is set to increase from $20^{\circ} \mathrm{C}$ to $90^{\circ} \mathrm{C}$ with a step of $10^{\circ} \mathrm{C}$. Due to the difference of the thermal coefficients between the core mode and the cladding mode, the wavelength of the resonant dip is red shifted when the temperature increases, as shown in Fig. 9. The temperature sensitivity of the proposed SSC-LPFG is $24.52 \mathrm{pm} /{ }^{\circ} \mathrm{C}$ with a linear correlation coefficient of 0.97339 . The temperature cross sensitivity of the liquid level is about $3.46 \mu \mathrm{m} /{ }^{\circ} \mathrm{C}$. The resolution of the liquid level measurements is $2.82 \mu \mathrm{m}$, and the temperature resolution is $0.82^{\circ} \mathrm{C}$. It reveals that a stable operation in the proposed optical sensor can be obtained.

The experimental results are compared with other references in Table 1. It shows that most of the fiber optic sensors with high liquid level sensitivity are prepared with interferometer structures. Generally, the measurement range occupies

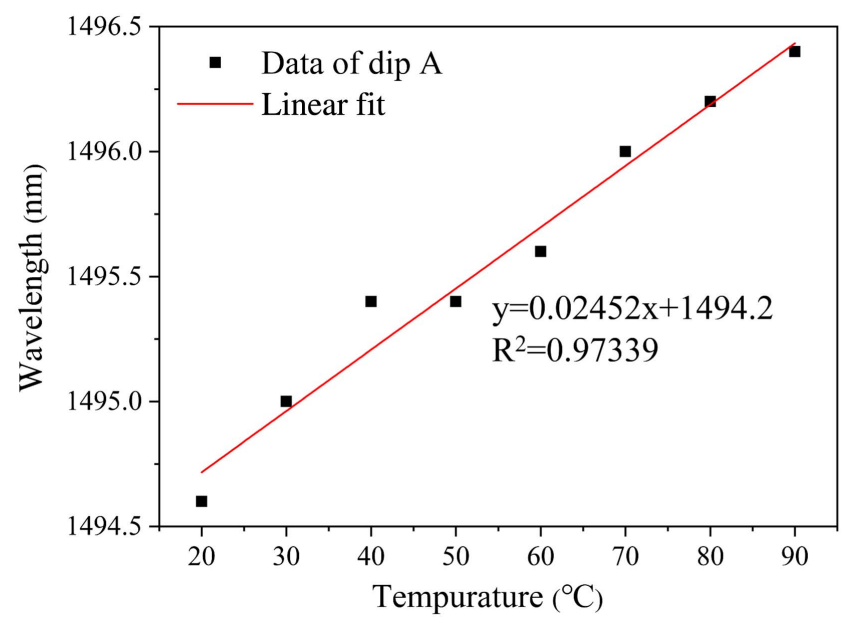

Fig. 9. Response of dip wavelength to temperature changing. 
Table 1. Parameter Comparison of Optical Fiber Liquid Level Sensors.

\begin{tabular}{|c|c|c|c|c|}
\hline Configuration & $\begin{array}{l}\text { Grating Length } \\
{[\mathrm{mm}]}\end{array}$ & $\begin{array}{l}\text { Level Range } \\
{[\mathrm{mm}]}\end{array}$ & $\begin{array}{l}\text { Level Sensitivity } \\
{[\mathrm{pm} / \mathrm{mm}]}\end{array}$ & $\begin{array}{l}\text { Temperature Crosstalk } \\
{\left[\mu \mathrm{m} /{ }^{\circ} \mathrm{C}\right]}\end{array}$ \\
\hline SMF-MMF-HCF-SMF/FBG with aligned spliced ${ }^{[21]}$ & 22 & 10 & 1145 (1.333 RI) & 7.86 \\
\hline SRI-based etched chirped FBG ${ }^{[22]}$ & 7 & 5.6 & 1214 & 6.5 \\
\hline SMF-taper-TCF-taper-SMF ${ }^{[23]}$ & 16 & 15 & 1241.6 & 17 \\
\hline SMF-PCF-SMF with a bending cantilever setup ${ }^{[28]}$ & Not reported & 8 & 111.27 (1.333 RI) & Not reported \\
\hline SMF-PMF-SMF with waist enlarged fiber tapers ${ }^{[29]}$ & 35 & 28 & 279 & 2.21 \\
\hline SMF-PMF-SMF with waist enlarged fiber tapers ${ }^{[29]}$ & 40 & 28 & 186 & Not reported \\
\hline SMF-MMF-TF-SMF with aligned spliced ${ }^{[30]}$ & 18 & 9 & -175.8 (1.334 RI) & 350 \\
\hline SMF-RCF-MMF-SMF with misaligned spliced ${ }^{[31]}$ & 300 & $१ २ ०$ & 6 & 6533 \\
\hline LLS based on a single LPFG ${ }^{[32]}$ & Not reported & 50 & 70 & Not reported \\
\hline This work & 3 & 1.4 & 7080 (1.333 RI) & 3.46 \\
\hline
\end{tabular}

$30 \%-70 \%$ of the sensor length. The length of the sensor is related to its sensitivity. The SSC-LPFG proposed in this paper sharply improves the sensitivity of the liquid level sensing while ensuring the mechanical performance. In addition, the SSC-LPFG structure has a low temperature response, which helps to reduce the temperature cross sensitivity in liquid level measurement. In terms of size comparison, the length range of the liquid level sensors based on gratings and interferometers is from 10 to tens of millimeters. The SSC-LPFG structure with a length of $3 \mathrm{~mm}$ has a strong competitive advantage in terms of integration. The liquid level sensing sensitivity of $7080 \mathrm{pm} / \mathrm{mm}$ provides a feasible solution for high-precision liquid level sensing required in medical and other fields.

\section{Conclusion}

In conclusion, a sensor based on SSC-LPFG for liquid level sensing is proposed. The proposed sensor is obtained by splicing MMFs with the SMF to get the C-MSM structure, and then it is polished on two opposite sides, which are parallel to the axis direction of the fiber. The sensitivity of the cladding mode to the external environment is improved by the insertion of multiple MMFs and double-sided polishing modulation by a high-power $\mathrm{CO}_{2}$ laser. The software simulation and experimental verification are carried out. In the experiment, the wavelength changes of the resonant dip are linearly related to the liquid level, and the corresponding average sensitivity to the liquid $(\mathrm{RI}=1.333)$ is $7080 \mathrm{pm} / \mathrm{mm}$. A temperature test is performed to verify the sensor's temperature sensitivity of $24.52 \mathrm{pm} /{ }^{\circ} \mathrm{C}$ and the cross sensitivity of $3.5 \mu \mathrm{m} /{ }^{\circ} \mathrm{C}$. The sensor has the advantages of compact structure and ultra-high sensitivity. It is a latent candidate in the field of high-precision liquid level measurement or sensing.

\section{Acknowledgement}

This work was supported by the Joint Research Fund in Astronomy under cooperative agreement between the National Natural Science Foundation of China (NSFC) and Chinese Academy of Sciences (CAS) (Nos. U1831115, U2031132, U1931206, and U2031130), Natural Science Foundation of Heilongjiang Province (No. ZD2019H003), and Fundamental Research Funds for the Central Universities to the Harbin Engineering University.

\section{References}

1. J. Villatoro, O. Arrizabalaga, G. Durana, I. S. de Ocariz, E. Antonio-Lopez, J. Zubia, A. Schulzgen, and R. Amezcua-Correa, "Accurate strain sensing based on super-mode interference in strongly coupled multi-core optical fibres," Sci. Rep. 7, 4451 (2017).

2. Z. Wang, L. Huang, C. Liu, H. Wang, and D. Yang, "Sensitivity-enhanced fiber temperature sensor based on Vernier effect and dual in-line MachZehnder interferometers," IEEE Sens. J. 19, 7983 (2019).

3. F. Pang, H. Liu, H. Guo, Y. Liu, and T. Wang, "In-fiber Mach-Zehnder interferometer based on double cladding fibers for refractive index sensor," IEEE Sens. J. 11, 2395 (2011).

4. H. Gong, Y. Xiao, N. Kai, C. L. Zhao, and X. Dong, "An optical fiber curvature sensor based on two peanut-shape structures modal interferometer," IEEE Photon. Technol. Lett. 26, 22 (2014).

5. B. Wang, W. Zhang, Z. Bai, L. Zhang, T. Yan, L. Chen, and Q. Zhou, "MachZehnder interferometer based on interference of selective high-order core modes," IEEE Photon. Technol. Lett. 28, 71 (2016).

6. C. He, C. Zhou, Q. Zhou, S. Xie, M. Xiao, J. Tian, and Y. Yao, "Simultaneous measurement of strain and temperature using Fabry-Pérot interferometry and antiresonant mechanism in a hollow-core fiber," Chin. Opt. Lett. 19, 041201 (2021).

7. J. E. Antonio-Lopez, J. J. Sanchez-Mondragon, P. Likamwa, and D. A. MayArrioja, "Fiber-optic sensor for liquid level measurement," Opt. Lett. 36, 3425 (2011).

8. Z. Song, Y. Li, J. Wei, and J. Hu, "Optical fiber torsion sensor based on pretwisted polarization-maintaining fiber Sagnac loop mirror structure," Laser Optoelectron. Prog. 58, 1706007 (2021). 
9. F. Baldini, M. Brenci, F. Chiavaioli, A. Giannetti, and C. Trono, "Optical fibre gratings as tools for chemical and biochemical sensing," Anal. Bioanal. Chem. 402, 109 (2012).

10. C. A. F. Marques, A. Pospori, D. Sáez-Rodríguez, K. Nielsen, O. Bang, and D. J. Webb, "Fuel level sensor based on polymer optical fiber Bragg gratings for aircraft applications," Int. Soc. Opt. Photon. 1674, 5759 (2016).

11. F. Li, N. Zhang, D. Xu, W. Niu, and B. Li, "Design of liquid level sensor of small range and multi-medium based on infrared correlation light curtain technology," Environ. Technol. 1004, 7204 (2016).

12. Y. Xu and Z. Wang, "The study and implementation of connected liquid level detection technology applying to medical equipment," in IEEE Workshop on Electronics, Computer and Applications (2014), p. 333.

13. I. K. Ilev and R. W. Waynant, "All-fiber-optic sensor for liquid level measurement,” Rev. Sci. Instrum. 70, 2551 (1999).

14. T. Guo, Q. Zhao, Q. Dou, Z. Hao, L. Xue, G. Huang, and X. Dong, "Temperature-insensitive fiber Bragg grating liquid-level sensor based on bending cantilever beam,” IEEE Photon. Technol. Lett. 17, 2400 (2005).

15. K. R. Sohn and J. H. Shim, "Liquid-level monitoring sensor systems using fiber Bragg grating embedded in cantilever," Sens. Actuator A 152, 248 (2009).

16. H. Fu, X. Shu, A. Zhang, W. Liu, and I. Bennion, "Implementation and characterization of liquid-level sensor based on a long-period fiber grating Mach-Zehnder interferometer," IEEE Sens. J. 11, 2878 (2011).

17. Y. Huang, B. Chen, G. Chen, H. Xiao, and S. U. Khan, "Simultaneous detection of liquid level and refractive index with a long-period fiber grating based sensor device," Meas. Sci. Technol. 24, 095303 (2013).

18. Q. Rong, X. Qiao, Y. Du, S. Hao, D. Feng, R. Wang, M. Hu, and Z. Feng, "Infiber quasi-Michelson interferometer for liquid level measurement with a core-cladding-modes fiber end-face mirror," Opt. Lasers Eng. 57, 53 (2014).

19. C. Rodríguez, M. Ribeiro, A. Frizera-Neto, C. Castellani, and M. J. Pontes, "Envelope-based technique for liquid level sensors using an in-line fiber Mach-Zehnder interferometer," Appl. Opt. 55, 9803 (2016).

20. Y. Jin, X. Dong, and M. Sun, “All-fiber Mach-Zehnder interferometer for liquid level measurement,” IEEE Sens. J. 15, 3984 (2015).
21. Y. Zhang, C. Weigang, Y. Lei, S. Wang, and T. Yan, "High sensitivity optical fiber liquid level sensor based on a compact MMF-HCF-FBG structure," Meas. Sci. 29, 055104 (2018)

22. H. Y. Chang, Y. C. Chang, H. J. Sheng, M. Y. Fu, W. F. Liu, and R. Kashyap, "An ultra-sensitive liquid-level indicator based on an etched chirped-fiber Bragg grating," IEEE Photon. Technol. Lett. 28, 268 (2015).

23. M. Shao, L. Han, J. Liang, R. Zhang, and H. Gao, "A high sensitivity liquid level sensor based on single mode-taper-thin core-taper-single mode fiber structure," Meas. Sci. 31, 105101 (2020)

24. S. Zhang, S. Deng, Z. Wang, W. Yang, C. Sun, X. Chen, Y. Ma, Y. Li, T. Geng, W. Sun, and L. Yuan, "Optimization and experiment of a miniature multimode fiber induced-LPG refractometer," OSA Continuum. 2, 2190 (2019).

25. S. W. James and R. P. Tatam, "Optical fibre long-period grating sensors: characteristics and application," Meas. Sci. 14, 49 (2003).

26. O. Duhem, J. F. Henninot, M. Warenghem, and M. Douay, "Demonstration of long-period-grating efficient couplings with an external medium of a refractive index higher than that of silica," Appl. Opt. 37, 7223 (1998).

27. H. J. Patrick and A. D. Kersey, "Analysis of the response of long period fiber gratings to external index of refraction," J. Lightwave Technol. 16, 1606 (1998).

28. X. Zhang, W. Peng, Z. Liu, and Z. Gong, "Fiber optic liquid level sensor based on integration of lever principle and optical interferometry," IEEE Photon. J. 6, 6801108 (2014).

29. H. Gong, H. Song, S. Zhang, K. Ni, and X. Dong, "An optical liquid level sensor based on polarization-maintaining fiber modal interferometer," Sens. Actuator A 205, 204 (2014).

30. L. Li, L. Xia, Z. Xie, and D. Liu, "All-fiber Mach-Zehnder interferometers for sensing applications," Opt. Express 20, 11109 (2012).

31. C. Castellani, H. Ximenes, R. L. Silva, A. Frizera-Neto, M. Ribeiro, and M. J. Pontes, "Multi-parameter interferometric sensor based on a reduced diameter core axial offseted fiber," IEEE Photon. Technol. Lett. 29, 239 (2017).

32. B. M. Mao, J. Canning, G. Peng, and B. Zhou, "Liquid level measurement sensor using a long-period fiber grating," Proc. SPIE 8351, 83511N (2012). 